\title{
CORONAVIRUS ANXIETY AND EXHAUSTION AMONG POLISH FRONT-LINE HEALTHCARE WORKERS - THE MEDIATION EFFECT OF INSOMNIA
}

\section{LUKASZ BAKA}

Central Institute for Labour Protection - National Research Institute, Warsaw, Poland

Department of Work Psychology

\begin{abstract}
Objectives: The study aimed to investigate the direct and indirect - mediated through insomnia - effect of coronavirus anxiety on exhaustion, from the perspective of Hobfoll's theory of conservation of resources (COR). According to the COR theory, critical events (e.g., the coronavirus epidemic) make people fearful of losing their valuable resources. A prolonged state of anxiety may lead to sleeping troubles, which over time results in an increase in exhaustion. Material and Methods: Data were collected from 440 Polish healthcare providers, including nurses and midwives, doctors, paramedics, medical assistance workers, and wardens. Three measures were used: the Coronavirus Anxiety Scale, the Copenhagen Psychosocial Questionnaire (the sleeping trouble subscale) and the Oldenburg Burnout Inventory (the exhaustion subscale). Hypotheses were tested using structural equation modeling. Results: The obtained results fully support the hypotheses. Both the direct and indirect relationships between coronavirus anxiety and exhaustion were observed. Specifically, high coronavirus anxiety increased insomnia, which in turn contributed to the development of exhaustion. Conclusions: The results are consistent with the COR theory. Prolonged coronavirus anxiety and sleeping problems depleted healthcare providers' resources and made them feel exhausted. Exhaustion among these workers can have serious consequences not only for themselves but also for the health of their patients. Therefore, research into effective ways to deal with coronavirus anxiety is needed. Int J Occup Med Environ Health. 2021;34(2):263-73
\end{abstract}

Key words:

insomnia, exhaustion, job burnout, conservation of resources theory, healthcare workers, coronavirus anxiety

\section{INTRODUCTION}

The COVID-19 pandemic caused by the spread of the SARS-CoV-2 coronavirus has changed the "world of work" and confronted it with new, previously unknown problems and challenges. In addition to concerns related to restructuring, job loss, the need to retrain, and modern technologies, the time of the pandemic caused employees to worry about their own health and life, as well as about their family members and relatives. The medical staff providing front- line healthcare during the epidemic constitute an occupational group highly exposed to the risk of coronavirus infection [1-3]. They face a great amount of job-related stressors including insufficient equipment, inadequate protection from contamination, high workload, frustration, discrimination, isolation, patients with negative emotions, and a lack of contact with their families [4,5]. The direct contact with infected patients and harsh working conditions are a source of everyday anxiety and strain for them $[5,6]$.

Funding: this work was supported by the National Centre for Research and Development (project No. IV.PB.03 entitled "Monitoring of psychosocial working conditions, work ability and well-being in a group of Polish employees," project manager: Łukasz Baka, Ph.D.).

Received: October 6, 2020. Accepted: February 11, 2021.

Corresponding author: Łukasz Baka, Central Institute for Labour Protection - National Research Institute, Department of Work Psychology, Czerniakowska 16, 00-701 Warsaw, Poland (e-mail: lubak@ciop.pl). 
Prolonged coronavirus anxiety can contribute to many mental health problems such as sleep disorders, stress or depressive symptoms [6], which over time result in exhaustion, perceived as the core of job burnout [7]. Exhaustion has seriously deteriorated medical professionals' performance of tasks involving attention, cognition, and memory, leading to medical errors in the battle against COVID-19 [8]. Thus, it also affects the health of patients. Several systematic literature reviews have found that high levels of job burnout in healthcare professionals are associated with less safe patient care $[9,10]$.

Drawing on the conservation of resources (COR) theory [11], the aim of the study is to test the direct and indirect (mediated via insomnia) relationship between coronavirus anxiety and exhaustion among Polish front-line healthcare workers. The link between general anxiety and job burnout is documented very well [12], also in the group of healthcare workers [13]. In one of the most recent metaanalyzes, this association was determined at $r=0.46$ [13]. However, to the best of the author's knowledge, there has been no previous study on the relationship between coronavirus anxiety and job burnout. This study seems to be important, especially in the group of healthcare workers who are burdened with a higher risk of infection.

\section{Coronavirus anxiety and exhaustion} among healthcare workers

According to the COR theory, "individuals strive to obtain, retain, and protect those things (resources) they most value, both material and psychosocial" [11]. These resources enable individuals, as well as their family and relatives, to survive; therefore, they feel stressed when threatened with resource loss, or when they actually lose resources or fail to regain resources following resource investment. People's fear of losing their own resources becomes especially visible in critical situations, e.g., during economic crises, natural or technological disasters, wars, or epidemics [14]. For example, some researchers have noted that resource loss was the most important predictor of psychological distress following a natural disaster $[15,16]$. The COVID-19 pandemic is an example of a resource-loss emergency. By October 4, 2020, nearly 35 million cases of COVID-19 had been reported in 188 countries and territories, including over 1 million deaths and over 24 million cases of recovery [17]. The pandemic caused major social and economic disruptions, including a severe global recession, the cancellation of major sporting, religious, political and cultural events, travel restrictions, and widespread supply shortages.

Based on recent studies, the pandemic is expected to have a substantial psychological impact, particularly on healthcare providers $[1,2]$. They comprise a notable proportion of the people who have actually contracted the illness. According to some reports, $10 \%$ of the confirmed cases of COVID-19 have involved medical staff [18]. In Poland, the rates are similar, with $9.79 \%$ of infected persons being healthcare providers (mainly doctors, nurses, midwives, and paramedics). This high rate of infection and mortality has a tremendous impact on healthcare workers' well-being. The deadly and uncontrolled nature of COVID-19, for which no effective treatment is known, along with relatively high infection rates and mortality among medical personnel, can provoke the feeling of anxiety and stress in this group [19]. Additional questions such as social stigmatization, the shortage of staff and personal protection equipment, poor working conditions, as well as a lack of consistent information about the spread of the virus, its contagiousness, effectiveness and prevention, can aggravate this situation.

The state of uncertainty and the lack of control over the source of anxiety increase its cognitive availability, which makes it easy to activate [20] and, with passing time, it becomes a chronic source of stress [21]. The COR theory points out that chronic exposure to resource loss (actual or expected) results in a gradual depletion of an individual's resource pool (called a loss cycle), and also that the loss of resources in 1 domain (e.g., an impact of coronavirus anxiety on general well-being) can exacerbate resource depletion in 
other areas (e.g., work-related well-being) [11]. Research has shown that the gradual depletion of general resources contributes to exhaustion (emotional, physical, and cognitive), that is treated as the basic component of job burnout [7]. Following the COR theory, it is argued that people feel exhausted when they perceive a continuous net loss of energetic coping resources that cannot be replenished [22]. $\mathrm{H1}$ : Coronavirus anxiety is positively related to exhaustion.

\section{Mediating role of insomnia}

Although coronavirus anxiety may lead to the gradual depletion of energy-related resources and exhaustion [11], the mechanisms that drive this process are not clear and have not been studied thus far. One potential mediation factor is insomnia, which is defined as difficulty in falling asleep and/or maintaining sleep [23]. Meta-analyses of studies have indicated that insomnia is the result of many factors, including stress $[24,25]$, anxiety $[26,27]$ and exposure to traumatic events [28,29]. More recent studies have pointed to coronavirus anxiety as a reason for insomnia [6,30]. There are at least 3 mechanisms by which coronavirus anxiety may lead to sleep disorders. First, this anxiety activates autonomic arousal and other strong negative emotions, all of which may affect increased insomnia [29,31,32]. Anxiety is also associated with low-grade inflammation (C-reactive protein) [33], which is related to difficulties in initiating sleep as well as with non-restorative sleep. Finally, coronavirus anxiety can also lead to intrusive and distressing thoughts (called ruminations) focused on negative past events and fears about the future [34], which can make it difficult to fall asleep and disturb sleep [35]. Huang and Zhao [36] reported that, during the COVID-19 outbreak, the insomnia problem was the most serious among healthcare workers compared to other occupational groups.

Prolonged insomnia depletes physical strength and mental energy of the individual, which over time results in a loss of energy, and a lack of physical and mental strength $[34,35]$.
As noted by Armon et al. [37], insomnia "may reduce resources for coping with stress, exacerbate symptoms of mental and physical fatigue among those who are already burned out, and lead to sustained burnout or the development of new cases of burnout." Indeed, several studies have supported the important role of insomnia in the development of burnout [38-40]. Therefore, it can be expected that insomnia may serve as a path by which coronavirus anxiety leads to exhaustion.

H2: Insomnia mediates the link between coronavirus anxiety and exhaustion

\section{MATERIAL AND METHODS \\ Subject}

The participants of the study were Polish healthcare workers $(\mathrm{N}=440)$ from 31 medical institutions (mainly hospitals and medical clinics) throughout Poland. In regards to their occupation, the most numerous were nurses and midwives $(\mathrm{N}=177)$, doctors $(\mathrm{N}=112)$, paramedics $(\mathrm{N}=$ $45)$, medical assistance workers $(\mathrm{N}=33)$, and wardens $(\mathrm{N}=28)$. The research group consisted of $353(80 \%)$ women and $87(20 \%)$ men, aged 20-71 years $(\mathrm{M} \pm \mathrm{SD}$ $43.81 \pm 11.13)$. Their work experience ranged $1-50$ years $(\mathrm{M} \pm \mathrm{SD} 19.50 \pm 10.99)$.

The choice of this professional group was dictated by the particularly burdensome working conditions of medical staff during the pandemic and their direct exposure to the risk of coronavirus infection. Additionally, while carrying out this research, the levels of coronavirus anxiety, insomnia and exhaustion in 3 occupational groups were compared, i.e., among healthcare workers, teachers and service workers having direct contacts with clients. It turned out that healthcare workers actually had the highest rates of coronavirus anxiety $(\mathrm{F}=2.34, \mathrm{p}<0.05)$ and job burnout $(\mathrm{F}=2.66, \mathrm{p}<$ 0.01 ), but not insomnia, compared to the other 2 groups.

The research was conducted in May-August 2020 by trained interviewers from a professional survey company with permission to enter the premises of the institution 
under study. Participation in the research was voluntary. Potential respondents received a hard copy of the questionnaires along with a letter explaining the purpose of the study. Full confidentiality of data and anonymity were guaranteed. The participants were asked to fill out the questionnaires and seal them in envelopes, which were subsequently collected by interviewers. All the participants were treated in accordance with the ethical guidelines of the Helsinki Declaration.

\section{Measures}

Three measures were used in the investigation.

\section{Coronavirus anxiety}

Coronavirus anxiety was measured with the Polish version of the Coronavirus Anxiety Scale (CAS) [41]. It is a brief self-reported mental health screener of dysfunctional anxiety associated with the coronavirus crisis, which consists of 5 items related to a variety of physical and mental ailments that appear in response to news or thoughts about the coronavirus (e.g., "I felt paralyzed or frozen when I thought about or was exposed to information about the coronavirus"). Each item contains answers from 0 ("not at all") to 4 ("nearly every day over the last 2 weeks"). In the present study, Cronbach's $\alpha$ and McDonald's $\omega$ coefficients for CAS were $\alpha=0.93$ and $\omega=0.93$, respectively.

\section{Insomnia}

Insomnia was measured with a subscale of the Polish version of the Copenhagen Psychosocial Questionnaire (COPSOQ II) [42,43] which refers to sleeping troubles, such as falling asleep and maintaining sleep, which occurred in the past 4 weeks. The subscale includes 4 questions (e.g., "How often have you slept badly or restlessly?") with a 5-point response scale ranging from 1 ("all the time") to 5 ("not at all"). Good statistical parameters of $\alpha=0.91$ and $\omega=0.92$ were observed in this study.

\section{Exhaustion}

Exhaustion was measured with the subscale of the Polish version of the Oldenburg Burnout Inventory [44,45]. The subscale consists of 8 items with a 5-point response scale ranging from 1 ("I completely disagree") to 5 ("I completely agree"), e.g., "After my work, I regularly feel worn out and weary." Good internal reliability was present with $\alpha=0.77$ and $\omega=0.79$ for exhaustion.

\section{Analytical procedure}

Prior to the verification of the 2 hypotheses, descriptive statistics were calculated, and a correlation analysis was carried out. In order to determine the factor accuracy and to estimate the parameters of fit, a confirmatory factor analysis (CFA) of the tools used in the structure proposed by its authors was also carried out. In the case of unacceptable measures of the model fit to data, the analysis of model parameters and modification indices was carried out in order to check the problem sources. Where it was possible to improve the parameters of the measurement model without interfering with the scale structure (e.g., without removing individual items), it was decided to introduce additional parameters to the model, i.e., covariances of measurement errors of the questionnaire items, and to reassess the parameters of fit for the measurement model - the root mean square error of approximation (RMSEA), the comparative fit index (CFI), the Tucker-Lewis index (TLI), and the standardized root mean square residual (SRMR).

After estimating the measurement models of the questionnaires, the internal consistency of the subscales was analyzed using coefficient $\omega$ which is more adequate for the analysis of latent factors than coefficient $\alpha$. Coefficients $\alpha$ were provided additionally, as these are more frequently used in statistical analyses. For the main part of the analysis, structural equation modeling was applied. Three variables including coronavirus anxiety, insomnia 
and exhaustion were introduced into the model. The following were tested:

- direct effects of coronavirus anxiety on exhaustion,

- indirect effects of insomnia on the link between coronavirus anxiety and exhaustion.

\section{RESULTS}

\section{Confirmatory factor analysis for the study variables}

In the first step, it was tested whether the proposed indices of coronavirus anxiety, insomnia and exhaustion had an assumed structure. In order to do so, a series of CFAs were conducted. The results are presented in Table 1. Prior to running CFA, all the data were screened for potential violation of normality, linearity, or seriousness, as well as an excessive number of outlying cases. No significant departures were found and CFA was continued. The model fit was judged based on standard and commonly used criteria. Values ranging $1.00-3.00$ for $\chi^{2}\left(\mathrm{CMIN} ; \chi^{2} / \mathrm{df}\right)$, values $>0.95$ for CFI, values $<0.06-0.08$ for RMSEA, values $<0.06-0.008$ for SRMR, and $p$ of close fit (PClose) $>0.05$ were understood as excellent parameters [46]. As an acceptable fit, CMIN ranging 3-5, RMSEA <0.08-0.10, PClose ranging $0.01-0.05$, SRMR ranging $0.08-0.10$, and
CFI ranging 0.90-0.95 were assumed. The non-significant coefficient for the $\chi^{2}$ test, which would indicate a good fit of the data to the model was not expected, because in general it is very difficult to achieve.

The CFA results have revealed excellent or acceptable parameters of the model fit. Each time, after assessing the basic model, some re-specifications based on error covariances were added, thus improving the fit of the models. For each analyzed model, all regression weights were significant $(\mathrm{p}<0.001)$ and sufficiently high $(>0.28)$, according to the criteria proposed by Fidell and Tabachnick [47]. Overall, all assumptions about the structure of the proposed indices were confirmed.

Three concepts analyzed in the study are close in meaning and may overlap [12]. Therefore, additional analyzes were performed for a 3 -factor model combined of the 3 measures (related to coronavirus anxiety, insomnia and exhaustion) and for a 1-factor model including all the scales as 1 factor. The results show that the 2-factor model (but not the 1-factor model) obtained acceptable fit parameters and is adequate (Table 1). These results confirm the distinctiveness of the theoretical constructs used in the study.

Table 1. Model adequacy and goodness of fit indices for 1- and 3-factor models and for the tools used in the study among Polish healthcare workers in 2020

\begin{tabular}{lccccccccc}
\hline \multicolumn{1}{c}{ Model } & $\chi^{2}$ & $\mathrm{df}$ & $\mathrm{p}$ & $\chi^{2} / \mathrm{df}$ & RMSEA & PClose & SRMR & CFI & AIC \\
\hline Coronavirus anxiety model & 59.64 & 9 & $<0.001$ & 6,62 & 0.09 & 0.002 & 0.07 & 0.91 & 12666.47 \\
$\quad$ with modifications & 30.32 & 8 & $<0.001$ & 3.79 & 0.06 & 0.094 & 0.05 & 0.95 & 11557.15 \\
Insomnia model & 31.94 & 7 & $<0.001$ & 4.56 & 0.08 & 0.128 & 0.08 & 0.88 & 11657.99 \\
$\quad$ with modifications & 19.81 & 6 & $<0.001$ & 3.30 & 0.04 & 0.313 & 0.03 & 0.93 & 10551.81 \\
Exhaustion model & 107.78 & 14 & $<0.001$ & 7.69 & 0.05 & 0.090 & 0.04 & 0.90 & 14975.76 \\
$\quad$ with modifications & 51.74 & 12 & $<0.001$ & 4.31 & 0.02 & 0.196 & 0.02 & 0.99 & 12663.74 \\
1-factor model & 2098.44 & 119 & $<0.001$ & 17.633 & 0.20 & 0.022 & 0.16 & 0.54 & 16463.70 \\
3-factor model & 600.377 & 116 & $<0.001$ & 5.175 & 0.08 & 0.174 & 0.07 & 0.90 & 14971.64 \\
\hline
\end{tabular}

AIC - Akaike information criterion; CFI - comparative fit index; PClose - $p$ of close fit; RMSEA - root mean square error of approximation; SRMR - standardized root mean square residual.

The re-specifications of models were achieved based on error covariance modification indices. 
Table 2. Descriptive statistics and correlation coefficients (Pearson's r) between variables in the study conducted among Polish healthcare workers in 2020

\begin{tabular}{lccccccc}
\hline \multirow{2}{*}{ Variable } & \multicolumn{9}{c}{ Pearson's correlation } & M & SD \\
\cline { 2 - 6 } & 1 & 2 & 3 & 4 & 5 & & \\
\hline 1. Age & - & & & & & 43.81 & 11.13 \\
2. Gender & 0.03 & - & & & - & - \\
3. Job seniority & $0.92^{* * *}$ & -0.01 & - & & 19.50 & 10.99 \\
4. Coronavirus anxiety & $0.18^{* * *}$ & $-0.17^{* *}$ & $0.13^{* *}$ & - & & 0.73 & 0.93 \\
5. Insomnia & $0.20^{* * *}$ & $-0.22^{* * *}$ & $0.18^{* * *}$ & $0.42^{* * *}$ & - & 3.84 & 0.89 \\
6. Exhaustion & $0.13^{* *}$ & $-0.14^{* *}$ & $0.12^{*}$ & $0.41^{* * *}$ & $0.39^{* * *}$ & 2.22 & 0.52 \\
\hline
\end{tabular}

${ }^{*} \mathrm{p}<0.05 ; * \mathrm{p}<0.01 ;{ }^{* * *} \mathrm{p}<0.001$

\section{Descriptive statistics}

After the examination of constructs validity, basic descriptive statistics for the study constructs were calculated (Table 2). The results of the correlational analysis confirmed the plausibility of the hypothesized model. Coronavirus anxiety is positively related to insomnia and exhaustion. The association between insomnia and exhaustion was supported, too. Additionally, the higher the age and job seniority of employees, the higher the levels of coronavirus anxiety, insomnia, and exhaustion. Gender differentiated the examined variables. The surveyed women experienced stronger coronavirus anxiety, had greater sleep problems, and felt more exhausted than the men.

\section{Main analysis - hypotheses verification}

The hypothesized model was examined by running structural equation modeling with Amos 25 equipped with additional plug-ins and estimands, e.g., "model fit measures" and "indirect effects" [48]. The results are presented in Figure 1 and Table 3. According to the results presented in Table 4, the hypothesized model had excellent values of fit indices in reference to the previously cited criteria (see above). Figure 1 shows the values of B-coefficients for the direct and indirect effects in the analyzed links. The data support a positive relationship between coronavirus anxiety and exhaustion. A partial mediation effect of insomnia was con- firmed, too, ab $=0.053, \mathrm{SE}=0.01, \mathrm{BCa} 95 \%$ CI: $0.03-0.08$, $p=0.001$. A higher level of coronavirus anxiety was related to higher insomnia, and this was related to a higher level of exhaustion. The results obtained confirm both $\mathrm{H} 1$ and $\mathrm{H} 2$.

\section{DISCUSSION}

The presented research aimed to explore the health effects of coronavirus anxiety. The effects of this anxiety on exhaustion (perceived as a key component of job burnout), both direct and mediated through insomnia, were tested [7]. The study group was composed of front-line healthcare workers. Some previous studies have shown that this occupational group is the most vulnerable to coronavirus infection $[2,8]$, and particularly susceptible to mental health problems, including sleeping troubles $[5,30,36]$ and job burnout $[1,19]$. One study showed that $27 \%$ of them had clinical levels of depression, $31 \%$ of anxiety, $34 \%$ of stress, $37 \%$ of post-traumatic stress, and $36 \%$ of job burnout [1].

The theoretical framework of the research was the COR theory [11]. According to the main assumption of the COR theory, the goal of human activity is to acquire, maintain and protect valuable and generally desired objects (the so-called resources). Stress occurs in circumstances related to the threat of losing, or actually losing, resources necessary for survival, and also when invest- 
ing a large pool of resources does not bring the expected result [11]. The risk of losing resources is particularly strong in crisis situations [14]; therefore, it was predicted that the outbreak of the pandemic triggered fear among medical workers about the risk of losing resources. Similar anxiety responses have been observed in medical staff during other emergencies, including SARS [49,50], natural disasters $[15,16]$ or a threat of terrorism [21]. Coronavirus anxiety was expected to lead, directly and indirectly by increasing sleep problems, to an increase in exhaustion.

It was expected that coronavirus anxiety would lead, directly and indirectly by increasing sleep problems, to an increase in exhaustion. The obtained research results confirmed these expectations. High coronavirus anxiety depleted healthcare providers' resources and made them feel exhausted. It was also observed that coronavirus anxiety increased insomnia, which in turn contributed to exhaustion among medical staff. Thus, both the direct and indirect effects of coronavirus anxiety were confirmed.

The results obtained are consistent with the COR theory [11], as well as with the results of previous studies on the health effects of coronavirus anxiety for healthcare workers $[5,9]$. Considering that exhausted medical workers are less involved in their work, tend to distance themselves from patients and professional duties, have low performance and make more mistakes, which may translate into patients' health [8-10], the obtained results have some important practical implications. Going forward, exhausted individuals may further exacerbate their losses by entering an escalating spiral of losses [22], especially since, as the COR theory predicts, the loss of resources curve is steeper than the corresponding

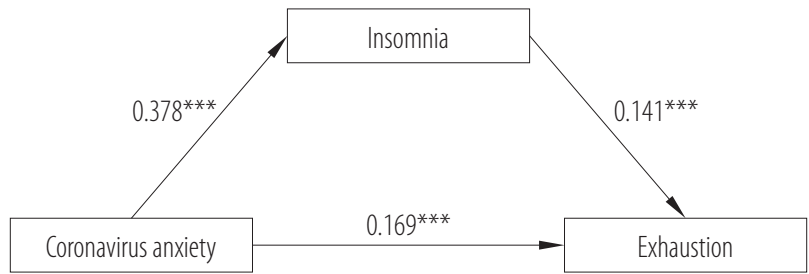

$* \mathrm{p}<0.05 ; * \mathrm{p}<0.01 ;{ }^{* * *} \mathrm{p}<0.001$.

Figure 1. The direct and indirect (mediated via insomnia) effect of coronavirus anxiety on exhaustion in the study among Polish healthcare workers in 2020

growth curve [11]. In other words, the pain and grief caused by the loss of a certain amount of one's own resources is usually stronger and more acute for a person than the joy of obtaining the same amount of the resources.

Some studies have confirmed the existence of a negative gain spiral between sleep disorders and job burnout [26,37]. It has also turned out that the level of anxiety about the coronavirus, as well as the level of insomnia and exhaustion, are high in older workers, with longer service, and especially in women. The strong anxiety of contracting a coronavirus infection in older workers may result from the fact that the detrimental effects of the virus and the mortality rate are the highest in this age group [51]. For example, a meta-analysis study with more than half a million of COVID-19 patients from China, Italy, Spain, United Kingdom and the New York State has indicated the determinant effect of age on mortality with the relevant thresholds of age $>50$ years, and especially $>60$ years [54].

The studies presented have some limitations. The key limitation refers to the cross-sectional design. Based on a single measurement of variables, it is difficult to iden-

Table 3. The parameters of adequacy and goodness of fit for the model tested using confirmatory factor analysis in the study conducted among Polish healthcare workers in 2020

\begin{tabular}{cccccccccc}
\hline Model & $\chi^{2}$ & $\mathrm{df}$ & $\mathrm{p}$ & $\chi^{2} / \mathrm{df}$ & RMSEA & PClose & SRMR & CFI & AIC \\
\hline Hypothesized model & 15.89 & 6 & 0.108 & 2.65 & 0.03 & 0.883 & 0.02 & 0.97 & 9267.09 \\
\hline
\end{tabular}

Abbreviations as in Table 1. 
tify a clear causal relationship between the analyzed variables [52]. No other conclusions than those which refer to the associations between the constructs measured at the same point of time can be made. The associations between variables (e.g., insomnia and exhaustion) may be of a reciprocal character. Future research should apply a longitudinal approach in order to clarify the direction of the relationships for coronavirus anxiety, insomnia, and exhaustion.

Moreover, as the research sample was limited to healthcare workers, any generalization to other occupational groups should be made with caution. Another limitation of the study was the unequal gender ratio in the sample, with $80 \%$ of female participants. The data may apply to men to a lesser extent. Any potential moderators which could modify the negative effect of coronavirus anxiety, such as job resources (e.g., social support) and personal resources (e.g., self-efficacy), were not included in the study. So, it is not known what organizational and personal factors make it possible to maintain "good" mental health, even in the conditions of strong exposure to the virus. Particularly useful would be those that relate to specific personal resources related to a job context (e.g., occupational hardiness or occupational resilience). As suggested by some authors, some specific job-related resources are particularly effective in reducing stress and maintaining "good" mental health [56]. It would be valuable to conduct such research in the future.

\section{CONCLUSIONS}

The obtained results supported the COR theory. Coronavirus anxiety resulting, to some extent, from an increased risk of infection among front-line healthcare workers was associated with a greater severity of mental health problems, such as sleeping troubles and exhaustion. Moreover, insomnia mediated the negative effect of coronavirus anxiety on exhaustion in this occupational group. Strong exhaustion probably resulted from the shortage of sufficient resources to coping, which had been compromised by prolonged stress and sleep deprivation. Some studies on the longitudinal detrimental impact of coronavirus anxiety on the mental health of medical staff, taking into account the moderating function of job and personal resources, are needed in the near future.

\section{REFERENCES}

1. Giusti EM, Pedroli E, D’Aniello GE, Stramba BC, Pietrabissa G, Manna C, et al. The Psychological Impact of the COVID-19 Outbreak on Health Professionals: A Cross-Sectional Study. Front Psychol. 2020;11:1664-84, https://doi.org/ 10.3389/fpsyg.2020.01684.

2. Pappa S, Ntellac V, Giannakasc T, Giannakoulisc V, Papoutsic E, Katsaounouc P. Prevalence of depression, anxiety, and insomnia among healthcare workers during the COVID-19 pandemic: A systematic review and meta-analysis. Brain Behav Immun. 2020;88:901-7, https://doi.org/10.1016/j.bbi.2020. 05.026

3. Nguyen LH, Drew DA, Graham MS, Joshi AD, ChuanGuo G, Wenjie M, et al. Risk of COVID-19 among front-line health-care workers and the general community: A prospective cohort study. Lancet. 2020;5:475-83, https://doi.org/10. 1016/S2468-2667(20)30164-X.

4. Fiorillo A, Gorwood P. The consequences of the COVID-19 pandemic on mental health and implications for clinical practice. Eur Psychiatr. 2020;63:12-4, https://doi.org/10.1192/j. eurpsy.2020.35.

5. Abdulah DM, Musa DH. Insomnia and stress of physicians during COVID-19 outbreak. Sleep Med. 2020;X2:100017, https://doi.org/10.1016/j.sleepx.2020.100017.

6. Lee SA, Mathis AA, Jobe MC. Mental health characteristics associated with dysfunctional Coronavirus Anxiety. Psychol Med. 2020;16:1-2, https://doi.org/10.1017/S0033291720 00121X.

7. Demerouti E, Bakker AB, Nachreiner F, Schaufeli WB. The job demands resources model of burnout. J Appl Psychol. 2001; 86:499-512, https://doi.org/10.1037//0021-9010.86.3.499. 
8. Fu W, Wang C, Zou L, Guo Y, Lu Z, Yan S. Psychological health, sleep quality, and coping styles to stress facing the COVID-19 in Wuhan, China. Trans Psych. 2020;10:225, https://doi.org/10.1038/s41398-020-00913-3.

9. Dewa CS, Loong D, Bonato S, Trojanowski L. The relationship between physician burnout and quality of healthcare in terms of safety and acceptability: a systematic review. BMJ Open. 2017;7:e015141, https://doi.org/10.1136/bmjopen-2016-015141.

10. Hall LH, Johnson J, Watt I, Tsipa A, O'Connor DB. Healthcare staff wellbeing, burnout, and patient safety: a systematic review. PLoS One. 2016;11(7):e0159015, https://doi.org/ 10.1371/journal.pone.0159015.

11. Hobfoll SE. Conservation of resources: A new attempt at conceptualizing stress. Am Psychol. 1989;44:513-24.

12. Koutsimani P, Montgomery A, Georganta K. The Relationship Between Burnout, Depression, and Anxiety: A Systematic Review and Meta-Analysis. Front Psychol. 2019;13(10): 284, https://doi.org/10.3389/fpsyg.2019.00284.

13. Turnipseed DL. Anxiety and burnout in the health care work environment. Psychol Rep. 1998;82:627-42, https://doi. org/10.2466/pr0.1998.82.2.627.

14. Kaniasty K. Klęska żywiołowa czy katastrofa społeczna. Psychospołeczne konsekwencje polskiej powodzi 1997 roku. Gdańsk: GWP; 2003.

15. Freddy JR, Shaw DL, Jarrell MP, Master CR. Towards an understanding of the psychological impact of natural disasters: An application of the conservation of resources stress model. J Trauma Stress. 1992;5:441-54.

16. Ironson G, Wynings C, Schneiderman N, Baum A, Rodriguez M, Greenwood D, et al. Posttraumatic stress symptoms, intrusive thoughts, loss and immune function after Hurricane Andrew. Psychosom Med. 1997;59:128-41.

17. Worldometer [Internet]. Delaware: Worldometer.info; 2021 [cited 2020 Oct 4]. Reported Cases and Deaths by Country or Territory. Available from: https://www.worldometers.info/ coronavirus/\#countries.

18. World Health Organization [Internet]. Geneva: In Memoriam; 2020 [cited 2020 Aug 7]. Healthcare Workers Who Have
Died of COVID-19. Available from: https://www.medscape. com/viewarticle/927976.

19. Mohammad J, Mahtab N, Fahimeh H, Kataun Z, Akbar F. Burnout among healthcare professionals during COVID-19 pandemic: A cross-sectional study. BMJ. Forthcoming 2021, https://doi.org/10.1101/2020.06.12.20129650.

20. Lerner JS, Keltner D. Fear, anger, and risk. J Pers Soc Psychol. 2001;81:146-59, https://doi.org/10.1037//0022-3514.81.1.146.

21. Toker S, Laurence G, Fried Y. Fear of terror and increased job burnout over time: Examining the mediating role of insomnia and the moderating role of work support. J Org Beh. 2015;36(2):272-91, https://doi.org/10.1002/job.1980.

22. Hobfoll SE, Shirom A. Conservation of Resources theory: applications to stress and management in the workplace. In: Golembiewski RT, editor. Handbook of organization behavior. New York: Dekker; 2000.

23. Lavie P. Sleep disturbances in the wake of traumatic events. N Engl J Med. 2001;345(25):1825-32, https://doi.org/10. 1056/NEJMra012893.

24. Ancoli-Israel S, Roth T. Characteristics of insomnia in the United States: results of the 1991 national sleep foundation survey. Sleep. 1999;22:347-53, https://doi.org/10.1111/j.14798425.2010.00481.x.

25. Healey ES, Kales A, Monroe LJ, Bixler EO, Chamberlin K, Soldatos CR. Onset of insomnia: role of life-stress events. Psychosom Med. 1981;43(5):439-51, https://doi.org/ 10.1097/00006842-198110000-00007.

26. Alvaro PK, Roberts RM, Harris JK. A Systematic Review Assessing Bidirectionality between Sleep Disturbances, Anxiety, and Depression. Sleep. 2013;36(7):1059-68, https:// doi.org/10.5665/sleep.2810.

27. Cox RC, Olatunji BO. Sleep in the anxiety-related disorders: A meta-analysis of subjective and objective research. Sleep Med Rev. 2020;51:101282, https://doi.org/10.1016/j.smrv. 2020.101282.

28. DiGrande L, Neria Y, Brackbill RM, Pulliam P, Galea S. Long-term posttraumatic stress symptoms among 3,271 civilian survivors of the September 11, 2001, terrorist attacks 
on the World Trade Center. Am J Epidemiol. 2011;1;173(3): 271-81, https://doi.org/10.1093/aje/kwq372.

29. Schuster MA, Stein BD, Jaycox LH, Collins RL, Marshall GN, Elliott MN. A national survey of stress reactions after the September 11, 2001 terrorist attacks. N Engl J Med. 2001;345(20):1507-12, https://doi.org/10.1056/NEJM200111 153452024.

30. Zhang C, Yang L, Liu S, Ma E, Wang Y, Cai Z, et al. Survey of Insomnia and Related Social Psychological Factors Among Medical Staff Involved in the 2019 Novel Coronavirus Disease Outbreak. Front Psychiatry. 2020;11:1-9, https:// doi.org/10.3389/fpsyt.2020.00306.

31. Bonnet MH, Arand DL. Hyperarousal and insomnia. Sleep Med Rev. 1997;2:97-108, https://doi.org/10.1016/S1087-0792 (97)90012-5.

32. Harvey AG. A cognitive model of insomnia. Behav Res Ther. 2002;40(8):869-93, https://doi.org/10.1016/S0005-7967 (01)00061-4.

33. Melamed S, Shirom A, Toker S, Berliner S, Shapira I. Association of fear of terror with low-grade inflammation among apparently healthy employed adults. Psychosom Med. 2004;66(4): 484-91, https://doi.org/10.1097/01.psy.0000130963.52755.b9.

34. Smith JM, Alloy LB. A roadmap to rumination: A review of the definition, assessment, and conceptualization of this multifaceted construct. Clin Psychol Rev. 2009;29(2):116-28, https://doi.org/10.1016/j.cpr.2008.10.003.

35. Carney CE, Harris AL, Moss TG, Edinger JD. Distinguishing rumination from worry in clinical insomnia. Behav Res Ther. 2010;48(6):540-6, https://doi.org/10.1016/j.brat.2010.03.004.

36. Huang Y, Zhao N. Generalized anxiety disorder, depressive symptoms and sleep quality during COVID-19 outbreak in China: a web-based cross-sectional survey. Psychiatry Res. 2020;288:112954, https://doi.org/10.1016/j.psychres.2020. 112954.

37. Armon G, Shirom A, Shapira I, Melamed S. On the nature of burnout-insomnia relationships: a prospective study of employed adults. J Psychosom Res. 2008;65(1):5-12, https:// doi.org/10.1016/j.jpsychores.2008.01.012.
38. Jansson-Fröjmark M, Lindblom K. Is there a bidirectional link between insomnia and burnout? A prospective study in the Swedish workforce. Int J Behav Med. 2010;17:306-13, https://doi.org/10.1007/s12529-010-9107-8.

39. Metlaine A, Sauvet F, Gomez-Merino D, Elbaz M, Delafosse JY, Leger D, et al. Association between insomnia symptoms, job strain and burnout syndrome: a cross-sectional survey of 1300 financial workers. BMJ Open. 2017;7(1):e012816, https://doi.org/10.1136/bmjopen-2016-012816.

40. Söderström M, Jeding K, Ekstedt M, Perski A, Akerstedt T. Insufficient sleep predicts clinical burnout. J Occup Health Psychol. 2012;17(2):175-83, https://doi.org/10.1037/a0027518.

41. Lee SA. Coronavirus Anxiety Scale: A brief mental health screener for COVID-19 related anxiety. Death Studies. 2020;44(7):393-401, https://doi.org/10.1080/07481187.2020. 1748481.

42. Pejtersen J, Kristensen T, Borg V, Bjorner J. The second version of the Copenhagen Psychosocial Questionnaire. Scand J Pub Health. 2010;38(3):8-24.

43. Baka L. Kopenhaski Kwestionariusz Psychospołeczny COPSOQ II. Podręcznik do polskiej wersji narzędzia. Warszawa: CIOP-PIB; 2019.

44. Demerouti E, Bakker AB, Vardakou I, Kantas A. The convergent validity of two burnout instruments. Eur J Psychol Assess. 2003;19:12-23, https://doi.org/10.1027//1015-5759. 19.1.12.

45. Baka L, Basińska B. Psychometric properties of Polish version of Oldenburgh Burnout Inventory. Med Pr. 2016;67(1): 29-41, https://doi.org/10.13075/mp.5893.00353.

46. Hu L, Bentler P. Cutoff criteria for fit indices in covariance structure analysis: conventional criteria versus new alternatives. Structural Equation Modeling. 1999;6:1-55.

47. Tabachnick BG, Fidell LS. Using multivariate statistics. 5th ed. London: Allyn \& Bacon/Pearson Education; 2007.

48. Gaskin J, Lim J. Model Fit Measures, AMOS Plugin. Gaskination's StatWiki; 2016.

49. Chen R, Chou K-R, Huang Y-J, Wang T-S, Liu S-Y, Ho L-Y. Effects of a SARS prevention programme in Taiwan on 
nursing staff's anxiety, depression and sleep quality: a longitudinal survey. Int J Nurs Stud. 2006;43:215e25.

50. Maunder RG, Lancee WJ, Balderson KE, Bennett J, Borgundvaag B, Evans S. Long-term psychological and occupational effects of providing hospital healthcare during SARS outbreak. Emerg Infect Dis. 2006;12:1924.

51. Bonanad C, García-Blas S, Tarazona-Santabalbina F, Sanchis J, Bertomeu-González V, Fácila L, et al. The effect of age on mortality in patients with COVID-19: A meta-analysis with 611,583 subjects. J Am Med Direct Assoc. 2020;21(7):915-8, https://doi.org/10.1016/j.jamda.2020.05.045.

52. De Jonge J, Dollard MF, Dormann C, Le Blanc PM, Houtman ILD. The demand-control model. Specific demands, specific control, and well-defined groups. Int J Stress Manag. 2000;7:269-87.

This work is available in Open Access model and licensed under a Creative Commons Attribution-NonCommercial 3.0 Poland License - http://creativecommons.org/ licenses/by-nc/3.0/pl/deed.en. 